Pacific Journal of Mathematics

HYPERCYCLIC RINGS

ILLIAM Howard CALDWELL 


\title{
HYPERCYCLIC RINGS
}

\author{
William H. Caldwell
}

The object of this paper is to provide characterizations for certain rings $R$ having the property that each cyclic right $R$-module has a cyclic injective hull. ${ }^{1}$ Such rings will be called hypercyclic. Characterizations for left perfect hypercyclic rings and commutative hypercyclic rings are given in terms of their ideal structure and self-injectivity. An example of a commutative hypercyclic ring without chain conditions is given to demonstrate that the characterization obtained is nontrivial.

In $\S 1$, the class of left perfect hypercyclic rings is shown to be precisely the class of uniserial rings. This is done by showing that they are quasi-Frobenius principal left ideal rings. In $\S 2$, commutative hypercyclic rings are shown to be semi-local, and then commutative local hypercyclic rings are described.

THEOREM. $R$ is commutative local hypercyclic if and only if either (a) $R$ is local uniserial or (b) $R$ satisfies the five conditions (i) The Jacobson radical is a nil ideal and is the union of an ascending sequence of proper principal subideals, (ii) Ideals in $R$ are linearly ordered, (iii) $R$ has nonzero socle, (iv) Nonprincipal ideals are of the form $x J, J=$ radical, (v) $R_{R}$ is injective.

The main result in $\S 1$ is a generalization of [3, Th. 2, p. 211].

1. Hypercyclic rings under chain conditions. We first require some definitions and conventions. First of all, we will reserve the letters $J$ and $S$ for the Jacobson radical and the socle, respectively, of $R$. We will denote the injective hull of $M_{R}$ by $E\left(M_{R}\right)$. It is well known that $E\left(M_{R}\right)$ is an essential extension of $M_{R}$, where $A_{R}$ essential in $B_{R}$ means that $A_{R} \cap C_{R} \neq 0$ for any $0 \neq C_{R} \subseteq B_{R}$. This will be denoted $A_{R} \subseteq{ }^{\prime} B_{R}$. In [2], Bass calls a ring left perfect provided every left $R$-module has a projective cover. $J$ is called left $T$-nilpotent provided for every sequence $\left\{a_{i}\right\}$ of elements of $J$ there exists an $n$ such that $a_{1} \cdot a_{2} \cdots a_{n}=0$. (Clearly a $T$-nilpotent radical is nil; however, we will later give an example of a ring which has a nil radical which is not $T$-nilpotent). Bass then gives the following equivalences for left perfectness.

${ }^{1}$ All rings are associative and have identity. Further, all modules $M_{R}$ are unital, i.e. $m \cdot 1=m$ for all $m \in M_{R}$. 
THEOREM P. The following are equivalent.

(1) $R$ is left perfect.

(2) $J$ is left $T$-nilpotent and $R / J$ is semi-simple.

(3) $R$ satisfies descending chain condition on principal right ideals.

(4) $R$ has no infinite sets of orthogonal idempotents and every nonzero right $R$-module has nonzero socle.

It is easy to see that any left or right artinian ring is right and left perfect, since a nilpotent radical is clearly $T$-nilpotent so that (2) is satisfied. More generally, any semi-primary ring is (both right and) left perfect. We borrow the following lemma from [3].

Lemma 1.1. A left perfect hypercyclic ring is right self injective.

Recall that a module $M$ is called a cogenerator for the class of right $R$-modules if $M$ satisfies the following property: If $A$ and $B$ are $R$-modules and $f: A_{R} \rightarrow B_{R}$ is a nonzero homomorphism then there exists $g: B_{R} \rightarrow M_{R}$ such that $g f$ is nonzero.

It is well known that an injective $R$-module $M$ is a cogenerator if and only if $M$ contains a copy of each simple right $R$-module. Consequently, a quasi-Frobenius ring is an injective cogenerator; in fact, it is well known that any left perfect right self injective ring is a cogenerator. Since a left perfect hypercyclic ring is right self injective by 1.1 , it is a cogenerator.

THEOREM 1.2. If $R$ is left perfect and right self injective, then any injective cyclic $R$-module is projective.

Proof. Since $R$ is perfect, $R / J$ is semi-simple, so

$$
R / J=\sum_{i=1}^{n} \oplus \bar{e}_{i}(R / J),
$$

$\left\{\bar{e}_{i}\right\}$ orthogonal idempotents and $\bar{e}_{i}(R / J)$ simple. Lift to a set $\left\{e_{i}\right\}$ of orthogonal idempotents of $R$ and write $R=\sum_{i=1}^{n} \oplus e_{i} R$. Hence each $e_{i} R$ is an indecomposable injective $R$-module. Since $R$ is perfect, $e_{i} R$ is the injective hull of its nonzero socle and hence must have simple socle $S_{i}$. Then $S=\sum_{i=1}^{n} \oplus S_{i}$ must be finite. We now show that the socle of any injective cyclic $R / I$ embeds in $R$. Then, since $R / I$ is essential over its socle, it will also embed in $R$, and being injective, be a summand of $R$, whence projective.

Let $C$ be a summand of the socle of $R / I$ maximal with respect to the property that $C$ embeds in $R$. Such a summand must exist 
since $S$ has finite length. Then there exists $D$ such that $C \oplus D=$ $S(R / I)$, the socle of $R / I$. We wish to show, then, that $D=0$.

Assume to the contrary. Then $D$ has a simple submodule $K$, and $K$ must embed in $R$ since $R$ is a cogenerator. Let $f: C \rightarrow R$ and $g: K \rightarrow R$ be the embeddings. Define $h: C \oplus K \rightarrow R \oplus R$ by $h((c, k))=$ $(f(c), g(k))$. Then $h$ is an embedding, and since $R \oplus R$ is injective, it must contain as a summand a copy of $E(C \oplus K)$, the injective hull of $C \oplus K$, so that $E(C \oplus K)$ is projective. Now $R / I$ also contains as a summand $E(C \oplus K)$, and if $\pi$ denotes the projection of $R / I$ onto $E(C \oplus K)$ and $\nu$ denotes the natural map of $R$ onto $R / I$ then $\pi \nu$ maps $R$ onto $E(C \oplus K)$. Since $E(C \oplus K)$ is projective, it is isomorphic with a summand of $R$, and since $C \oplus K \subseteq E(C \oplus K), C \oplus K$ embeds in $R$, contradicting the maximality of $C$. This contradiction establishes that $C=S(R / I)$, and, consequently, the theorem. Notice that any cyclic injective, then, embeds in $R$ in fact, this is what we proved.

If $I$ is any subset of $R$, we denote by $r(I)$ and $s(I)$ respectively the right and left annihilator of $I$ in $R$. That is, $r(I)=\{x \in R \mid I x=0\}$ and $s(I)=\{x \in R \mid x I=0\}$. If $I$ consists of the single element $x$, we will write $(0: x)$ for $r(I) .^{2}$

We need the following lemma from [5].

Lemma 1.3. If $R_{R}$ is injective, then finitely generated left ideals are left annihilators.

THEOREM 1.4. If $R$ is left perfect and hypercyclic, then annihilator left ideals are principal left ideals.

Proof. By Lemma 1.1, $R_{R}$ is injective, so that $R$ satisfies the conditions of Theorem 1.2. Consequently, every injective cyclic $R$ module embeds in $R$, so that, since every cyclic embeds in an injective cyclic (its injective hull), every cyclic embeds in $R$. Hence every right ideal is the right annihilator of a single element. Let $L$ be any left annihilator ideal, $L=s(I)$ for some right ideal $I$ in $R$. Then $r(L)=$ $I=(0: x)$ for some $x \in R$. Since $(0: x)=(0: R x)=r(R x), r(L)=r(R x)$. But $R$ is injective, so that principal left ideals are left annihilators and since $L$ is also a left annihilator, $L=s(r(L))=s(r(R x))=R x$.

THEOREM 1.5. $R$ is left perfect and hypercyclic if and only if $R$ is uniserial.

Proof. That any uniserial ring is left perfect and hypercyclic follows from [3, Th. 4.2] and the fact that any artinian ring is perfect

${ }^{2}$ More generally, if $A_{R} \leqq B_{R},(A: B)=\{r \in R \mid B r \leqq A\}$ 
Conversely, assume $R$ satisfies the conditions of the theorem. Then $R$ is an injective cogenerator, so that $J$ is the left annihilator of $S$ (see [8]). Hence, $J=R x$ for some $x \in R$ by 1.4. But $R$ is left perfect, so that $J$ is $T$-nilpotent, and consequently nil. Thus $x^{n}=0$ for some $n$. Since $J$ is an ideal, $J^{n}=(R x)^{n} \subseteq R x^{n}=0$, so that $J$ is nilpotent. But then $R$ is semi-primary, and so is also right perfect. It follows that $R$ satisfies descending chain condition for principal left ideals. Let $I_{0} \subseteq I_{1} \subseteq I_{2} \subseteq \cdots$ be an ascending chain of right ideals of $R$. Let $I_{k}=\left(0: x_{k}\right)$. Then $R x_{1} \supseteqq R x_{2} \supseteqq \cdots$ is a descending chain of left ideals of $R$ which must terminate, so there is an integer $k$ such that $R x_{k}=R x_{k+p}$ for every $p$. But then $I_{k}=I_{k+p}$ for every $p$ so $R$ is right neotherian. By [3, Th. 4.2] $R$ is uniserial.

2. Commutative hypercyclic rings. In $\S 1$ the first result derived from chain conditions was the injectivity of $R_{R}$. Commutativity is also a sufficient hypothesis to guarantee injectivity of a hypercyclic ring.

LEMMA 2.1. If $R$ is commutative and $R / A$ embeds in $R / B$ for any ideals $A, B \subseteq R$, then $B \subseteq A$.

Proof. If $f$ is the embedding and $b \in B$, then $f(b+A)=$ $f(1+A) b=0$.

CoRollary 2.2. A commutative hypercyclic ring is self-injective.

Proof. If $R / I \cong E(R), R$ embeds in $R / I$.

In [7], Osofsky proves the following three lemmas.

Lemma a. Let $\left\{e_{n} \mid n \in N\right\}$ be a set of orthogonal idempotents in a right self injective regular ring. Then for every subset $A \subseteq N$, there is an idempotent $E_{A} \in R$ such that:

$$
\begin{aligned}
E_{A} e_{n} & =e_{n} \quad \text { for all } n \in A \\
E_{A} e_{n^{\prime}} & =e_{n^{\prime}} E_{A}=0 \quad \text { for all } n^{\prime} \in N \sim A \\
E_{A}+E_{N \sim A} & =E_{N} .
\end{aligned}
$$

Lemma b. Let $R$ be a right-self injective regular ring containing an infinite set of orthogonal idempotents $\left\{e_{n} \mid n \in N\right\}$. Let $I=\sum_{n} \in_{N} e_{n} R$. For $A \subseteq N$, define $E_{A}$ as in Lemma a. Then the set $S_{\mathscr{A}}=\left\{E_{A} \mid A \in \mathscr{A}\right\}$, where each $A$ is infinite, is independent modulo $I$ if and only if for any finite set $\left\{A_{i} \mid i=1, \cdots, n\right\} \subseteq \mathscr{A}, A_{i} \cap \mathbf{U}_{j \neq i} A_{j}$ is a finite subset of $N, 1 \leqq i \leqq n$. 
Lemma c. Let $R$ be a right self injective regular ring which contains an infinite set of orthogonal idempotents $\left\{e_{n} \mid n \in N\right\}$. If $I=\sum_{n} \epsilon_{N} e_{n} R$, then $R / I$ is not an injective $R$-module.

Let $a^{\prime}, b^{\prime}, c^{\prime}$ designate respectively the lemmas resulting when the word "commutative" is substituted for "regular" in Lemmas a, b, and c. Then $\mathrm{a}^{\prime}, \mathrm{b}^{\prime}$ and $\mathrm{c}^{\prime}$ are also true. ${ }^{3}$

Proof. We first point out that the hypothesis of Lemma c are used in [7] only to guarantee that the conclusions of Lemmas a and $\mathrm{b}$ hold. Hence we need only show that $\mathrm{a}^{\prime}$ and $\mathrm{b}^{\prime}$ are true.

$\mathrm{a}^{\prime}$. Since $R$ is injective, $R / J$ as also injective (see (9)), and thus satisfies Lemma a. Let $\left\{\bar{E}_{A}\right\}$ designate the idempotents obtained from the set $\left\{\bar{e}_{n}\right\}$ of orthogonal idempotents in $R / J$. Lift to corresponding sets of orthogonal idempotents $\left\{E_{A}\right\}$ and $\left\{e_{n}\right\}$ in $R$.

By Lemma a, $E_{A} e_{n}-e_{n}=x \in J$. Then, $e_{n} x=x$, so that $E_{A} e_{n}=$ $e_{n}+x=e_{n}+e_{n} x=e_{n}(1+x)$. Hence $e_{n}=E_{A} e_{n}(1+x)^{-1} \in E_{A} R$ so $E_{A} e_{n}=e_{n}$. The second property in Lemma $a^{\prime}$ follows since $E_{A} e_{n^{\prime}}$ is idempotent and in $J$. The same argument as above shows that $E_{A}$ is orthogonal to $E_{N \sim A}$. Now since $E_{A}+E_{N \sim A}-E_{N}=K \in J E_{A}-E_{A} E_{N} \in J$, so that $E_{A}=E_{A} E_{N}$ as above. Hence $E_{A} K=E_{N \sim A} K=0$, and $E_{N} K=K$. Hence

$$
E_{N}+K=E_{N}+E_{N} K=E_{N}(1+K)=E_{A}+E_{N \sim A}
$$

so that $E_{N}=\left(E_{A}+E_{N \sim A}\right)(1-K)^{-1} \in\left(E_{A}+E_{N \sim A}\right) R$, so $0=\left(E_{A}+E_{N \sim A}\right) K=$ $\left(E_{A}+E_{N \sim A}\right) E_{N} K=E_{N} K=K$.

$b^{\prime}$. Let $S_{a}$ be independent modulo $I$. We know that Lemma $b$ applies to $R / J$, so we need only show that if $S_{a}$ is independent modulo $I$ then it is independent modulo $I+J$. However, it is easily seen that $\left\{E_{A}\right\}$ is independent modulo an ideal $K$ is and only if for every finite subset $\left\{E_{A_{i}} \mid i=1, \cdots, n\right\} \subseteq\left\{E_{A}\right\}, E_{A_{j}} \cap \sum_{i \neq j} E_{A_{i}} \subseteq K, j=1, \cdots, n$. Hence since $I \subseteq I+J$, independence $\bmod I$ implies independence $\bmod J$.

The converse is proved exactly as in [7].

We use $c^{\prime}$ to conclude that a commutative hypercyclic ring can have no infinite sets of orthogonal idempotents, and consequently must be semi-simple modulo its radical.

THEOREM 2.3. If $\left\{e_{i}\right\}$ is any set of idempotents in a commutative hypercyclic ring $R$, and if $I=\sum e_{i} R$, then $R / I$ is an injective $R$-module.

Proof. Let $E(R / I)=R / K$. Then $R / I$ embeds essentially in $R / K$, so $K \cong I$ by 1.1. Now if $e$ is any idempotent in $R$,

3 The author is indebted to Dr. Osofsky who pointed out this fact.

${ }^{4}$ This is possible in any self injective ring, see [10]. 


$$
R / K=\frac{(1-e) R+K}{K} \oplus \frac{e R+K}{K} .
$$

The sum is direct since $R$ is commutative. Let $\varphi: R / I \rightarrow R / K$ denote the embedding. Now for any $x \in R$, if $\varphi(x+I) \in(e R+K) / K$, write $\varphi(x+I)=y+K$. Then $y e+K=e y+K=y+K$, so $\varphi(x+I)=$ $\varphi(x+I) e$. Since $\varphi((x+I) e=\varphi(x e+I)=\varphi(e x+I)=\varphi(0+I)=0$ whenever $e \in I,(e R+K) / K \cap \varphi(R / I)=0$ whenever $e \in I$. Since $(R / I)$ is essential in $R / K, e R \subseteq K$. Hence $I \subseteq K$. So $I=K$. Hence $R / I$ is injective.

CoROLlary 2.4. If $R$ is a commutative hypercyclic ring, then $R / J$ is semi-simple.

Proof. Since $R$ is injective, $R / J$ is a right self injective regular ring, so that $R / J$ is semi-simple if and only if it has no infinite sets of orthogonal idempotents. Since orthogonal idempotents can be lifted orthogonally modulo $J, R / J$ is semi-simple if and only if $R$ has no infinite sets of orthogonal idempotents. However if $R$ has an infinite set of orthogonal idempotents, $\left\{e_{i}\right\}$, and if $I=\sum e_{i} R, R / I$ would be noninjective by Lemma $c^{\prime}$. This can't happen by the theorem.

Notation. We will call a commutative local hypercyclic ring a $\mathrm{CLH}$ ring.

THEOREM 2.5. A commutative ring $R$ is hypercyclic if and only if $R$ is a ring direct sum of a finite number of CLH rings.

Proof. Assume $R$ is hypercyclic. Since $R / J$ is semi-simple, $R$ is semi-local (a ring direct sum of a finite number of local rings). Now if $e R$ is a local component of $R$ and $M$ is an $R$-module, then $M(1-e)=0$ and so maps of ideals $I$ of $R$ into $M$ reduce to maps of ideals $e I$ into $M$ and so $R$-injectivity of $M$ is equivalent to $e R$ injectivity. Also, notice that $R / I$ is an $e R$ module if and only if $(1-e) R \subseteq I$, that is, if and only if $R / I$ is a cyclic $e R$-module. Since $R$ is hypercyclic, every cyclic $e R$-module $e R / e I$ has a cyclic $R$-injective hull, and since this must be $e R$-injective, the $e R$-injective hull of $e R / e I$ is a cyclic $e R$-module, so each $e R$ is a CLH ring. Conversely, let $R=\sum_{i=1}^{n} \oplus e_{i} R$, where each $e_{i} R$ is a CLH ring. Then if $I$ is any ideal in $R, I=\sum_{i=1}^{n} \oplus e_{i} I$, so that $R / I \cong \sum_{i=1}^{n} \oplus e_{i} R / e_{i} I$. Consequently $E(R / I) \cong \sum_{i=1}^{n} \oplus E\left(e_{i} R / e_{i} I\right)$. Again we notice that the $R$-injective hull coincides with the $e_{i} R$-injective hull of $e_{i} R / e_{i} I$. However each $e_{i} R$ is $\mathrm{CLH}$, so that the $e_{i} R$-injective hull of $e_{2} R / e_{i} I$ is isomorphic with $e_{i} R / A_{i}$ for some $A_{i} \cong e_{i} R$. Hence $E(R / I) \cong \sum_{i=1}^{n} \oplus e_{i} R / A_{i} \cong R / \sum_{i=1}^{n} \oplus A_{i}$ is cyclic and hence $R$ is hypercyclic. This completes the proof. By this theorem, we can obtain a characterization of commutative hypercyclic rings by restricting our attention to $\mathrm{CLH}$ rings. 
It has been shown that in the presence of certain chain conditions, hypercyclic rings are actually uniserial. For a uniserial ring every principal indecomposable left or right ideal has a unique composition series. We will show that CLH rings have a similar property, specifically, that ideals in such rings are linearly ordered. In [2], Bass calls a submodule $N$ of an $R$ module $M$ superfluous provided $A+N=M$ implies $A=M$ for all submodules $A$ of $M$. It is easy to show that the radical of a ring is a superfluous submodule of $R_{R}$ and that submodules of superfluous modules are superfluous.

LEMma 2.6. If $R$ is a commutative local ring, then cyclic $R$ modules are indecomposable.

Proof. If $R / A=C / A \oplus D / A$, then $C+D=R$, and since every ideal different from $R$ is contained in $J=\operatorname{rad} R$, either $C$ or $D$ is superfluous, so that $R=C$, say, hence $D \subseteq A$ and $R / A$ is indecomposable.

If $A$ is any submodule of an $R$-module $M$, a submodule $B$ of $M$ which is maximal with respect to the property that $A \cap B=0$ is called a complement of $A$ in $M$. Thus any non-ssential submodule of $M$ has nonzero complement in $M$. Clearly, if $B$ is a complement of $A$, then $A \oplus B \subseteq M$. If $M$ is also injective, then whenever $B$ is a complement of $A, M^{\prime} \cong E(A \oplus B)=E(A) \oplus E(B)$, so that $M$ is decomposable if $A$ and $B$ are both nonzero.

THEOREM 2.7. If $R$ is a CLH ring, then ideals in $R$ are linearly ordered.

Proof. Let $A$ and $B$ be ideals in $R$. If $A / A \cap B$ and $B / A \cap B$ were both nonzero, then $A / A \cap B$ would have nonzero complement in $R / A \cap B$. But then $A / A \cap B \oplus \operatorname{comp}(A / A \cap B)$ would lead to a decomposition of $E(R / A \cap B)$, and since the injective hull of $R / A \cap B$ is cyclic, it is indecomposable by Lemma 2.6. Hence $A / A \cap B$ or $B / A \cap B=0$, thus $A \subseteq A \cap B$ or $B \subseteq A \cap B$ hence, $A \subseteq B$ or $B \cong A$.

CoRollary 2.8. Finitely generated ideals in a $\mathrm{CLH}$ ring are principal.

Proof. Let $I=x_{1} R+\cdots+x_{n} R$. Then by the theorem, there exists an integer $i$ such that $x_{j} R \leqq x_{i} R, j=1, \cdots, n$. That is, $I=x_{i} R$.

Definition. $M_{R}$ is called faithful provided $M r=0$ if and only if $r=0 . \quad(M r=\{m r \mid m \in M\})$. 
In [8], the following lemma is proved, which we find useful.

Lemma 2.9. If $R$ is any ring and $\left\{S_{i} \mid i \in I\right\}$ a complete set of representatives of isomorphism classes of simple $R$-modules, then $\sum_{i \in I} \oplus E\left(S_{i}\right)$ is a faithful $R$-module.

Since clearly any module $M$ with a faithful submodule is itself faithful, in order to show that an $R$-module $M$ is faithful, it suffices to prove that $M$ contains a copy of the injective hull of each simple.

TheORem 2.10. If $R$ is a CLH ring, then $R$ has a simple socle.

Proof. By Theorem 2.7, we need only show that $S \neq 0$. Let $E(R / J)=R / K$. Then $R / K$ is faithful by Lemma 2.9 , so that $(R / K) K=$ 0 implies $K=0$, hence $R / J$ embeds in $R$ so $S \neq 0$.

Observe that $S$ is essential in $R$, since $R$ is indecomposable.

THEOREM 2.11. If $x R$ is a cyclic module over a CLH ring $R$ then any submodule of $x R$ is essential in $x R$.

Proof. Evident by 2.7.

THEOREM 2.12. Let $R$ be a CLH ring. Then for any ideal $I, R / I$ has nonzero socle if and only if $E(R / I)=R$.

Proof. If $E(R / I)=R$, then $R / I$ embeds in $R$. Since by Theorem 2.10, $S$ is simple and essential in $R$, it is contained in every ideal, hence, the image of $R / I$ has nonzero socle, so $R / I$ does.

Conversely, if $R / I$ has nonzero socle, then $E(R / I)$ contains a copy of the injective hull of the unique simple, and so is a faithful $R$-module, so that if $E(R / I) \cong R / K$, then $R / K$ is faithful. But $(R / K) \cdot K=0$ so that $K=0$.

It is well known that a module $M_{R}$ is injective if and only if for every right ideal $I$ in $R$ and each homomorphism $\varphi: I \rightarrow M$ there exists $m \in M$ such that $\varphi(r)=m r$ for every $r \in I$. This condition will be called Baer's condition in the ensuing, as it is in [4].

THEOREM 2.13. Let $R$ be a CLH ring, and let $I$ be an ideal of $R$ such that $(0: x) \subseteq I$ for some $x \in J$. Then $R / I$ is not injective unless $I=R$.

Proof. Assume $R / I$ injective, $(0: x) \subseteq I$. Define $\varphi: x R \rightarrow R / I$ by $\varphi(x r)=r+I$. Since $x r=0$ implies $r \in(0: x) \leqq I$, so that $r+I=0$, $\varphi$ is well defined. Clearly $\varphi$ is an $R$-homomorphism. Since $R / I$ is 
injective, by Baer's condition there exists $m \in R$ such that $\varphi(x r)=$ $(m+I) x r=m x r+I$ for all $x r \in x R$. But then $1+I=\varphi(x \cdot 1)=$ $m x+I$, so that $1-m x \in I$. Since $x \in J,(1-m x)^{-1} \in R$, so that $1=$ $(1-m x)(1-m x)^{-1} \in I$. Hence, $I=R$.

CoRollary 2.14. If $R$ is a $\mathrm{CLH}$ ring and if $J$ is principal, then $E(R / I) \cong R$ for each right ideal $I \neq R$.

Proof. Let $J=x R$. Then $S=(0: J)=(0: x)$ is contained in every nonzero ideal $I$, so that by the theorem, $R / I$ is not injective, hence, $R$ is the only injective cyclic.

COROLlary 2.15. If $R / S$ has nonzero socle for a CLH ring $R$, then $E(R / I) \cong R$ for each ideal $I \neq R$.

Proof. $R / S$ has nonzero socle, so $E(R / S) \cong R$ by Theorem 2.12, hence $R / S$ embeds in $R$. So $R / S \cong x R$ where $S=(0: x)$. Since $S=$ $(0: J),(0: x)=(0: R x)=(0: J)$. But $R_{R}$ is injective, so that principal ideals are annihilators by Lemma 1.3 , and since $J$ is maximal, it is an annihilator. Hence, $x R=(0:(0: x R))=(0:(0: J)=J$, so $J$ is principal. By Corollary 2.14, the proof is complete.

Lemma 2.16. Let $R$ be a commutative local ring, $0 \neq x \in R$. Then $x R / x J$ is a nonzero simple $R$-module.

Proof. Since $R /(0: x) \cong x R$ and

$$
(0: x) \subseteq J, R / J \cong R /(0: x) / J /(0: x) \cong x R / x J
$$

is simple.

THEOREM 2.17. Let $R$ be a $\mathrm{CLH}$ ring. If $I$ is an ideal in $R$ such that $(R / I)_{R}$ is injective, then $I=0$ or $I=S$.

Proof. We show that if $R \supsetneqq I \supsetneqq S$, then $R / I$ is not injective. Let $x \in I, x \notin S$. Since $x R \supsetneqq S$, there is an element $r \in R$ such that $0 \neq z=x r \in S$, and so $z R=S$. Further, since $x R \neq S$, and $x R / x J$ is simple by Lemma 2.16, $S \subseteq x J$, so that $r \in J$. But then $r(x J)=$ $x r J=z J \subseteq S J=0$, so that $x J \subseteq(0: r)$. If $x J \neq(0: r)$ then $x R \cong$ $(0: r)$ so $0 \neq z=x r=0$, a contradiction. By Theorem 2.13, since $x J \subseteq I, R / I$ is not injective.

We will show in a later example that it is possible for both $R_{R}$ and $(R / S)_{R}$ to be injective for a CLH ring $R$, however, we do not have enough information to do so now. 
The techniques of Theorem 2.17 allow us to describe exactly the ideals of a CLH ring. We first have a corollary to Theorem 2.17.

Corollary 2.18. Let $I$ be an ideal in a CLH ring $R$. If $I \neq$ $(0: x)$ for any $x \in R$, then there is an $x \in R$ such that $I=(S: x)$.

Proof. If $I \neq(0: x)$ for any $x \in R$, then $R / I$ does not embed in $R$, so that $E(R / I)$ is not isomorphic with $R$. By the theorem, $E(R / I)=$ $R / S$, so that $R / I$ embeds in $R / S$. If $f$ is the embedding, $I=(S: x)$ where $f(1+I)=x+S$.

THEOREM 2.19. Let $I$ be an ideal in a CLH ring $R$. Then $I$ is principal or $I=x J$ for some $x \in R$.

Proof. We show first that given any element $0 \neq y \in R$ there exists an element $z \in R$ such that $(S: y)=z R,(S: z)=y R,(0: y)=z J$, and $(0: z)=y J$. Since by Corollary 2.18 , every ideal of $R$ must be of the form $(0: x)$ or $(S: x)$ the proof will be complete.

Let $0 \neq y \in R$. Then $y R \supset S$, so there exists $z \in R$ such that $y z \neq 0$ is in $S$. Hence, $y z R=S$, and so $z R \subseteq(S: y)$. However, if $t \in(S: y)$ and $t \notin z R$, then $z R \subseteq t J$ so that there is $j \in J$ such that $z=t j$. Then $z y=y t j \in S J=0$, a contradiction. Hence $z R=(S: y)$. Since $y z R=z y R, y R \subseteq(S: z)$ and an argument symmetric to the above shows that $y R=(S: z)$. Now since $y z \in S, z J=(0: y)$ and $(0: z)=y J$.

Definition. An ideal $I$ in a ring $R$ is called nil provided every element of $I$ is nilpotent.

THeOREM 2.20. If $R$ is a CLH ring, then $J$ is nil.

Proof. Let $y \in J$, and let $I=\bigcap_{n=1}^{\infty} y^{n} R$. Then $I=0$ if and only if $y$ is nilpotent, for if $y^{n} \neq 0$ for every $n$, then $S \subseteq y^{n} R$ for every $n$, so $S \subseteq I$. We will show that the assumption that there is a $y \in J$ such that $I \neq 0$ leads to a contradiction. We first show that $y I=I$. If $I=0$, this is obvious, so assume $I \neq 0$. Then $y^{n} \notin I$ for any $n$, for if so, $y^{n} \in y^{n+1} R$ so $y^{n}=y^{n+1} r$ and hence,

$$
y^{n}=y^{n}(1-y r)(1-y r)^{-1}=\left(y^{n}-y^{n+1} r\right)(1-y r)^{-1}=0,
$$

a contradiction. Now let $t \in I$. Then $t=y r$ for some $r \in R$, and if $r \notin I$, there is some $n$ such that $r R \supseteqq y^{n} R$, so that $r k=y^{n}$ for some $k \in R$. Hence, $y^{n+1}=y r k=t k \in I$, a contradiction.

This shows that $r \in I$, so that $t \in y I$, and hence $y I=I$.

Now by Theorem 2.19, $I=x R$ or $x J$ for some $x \in R$. Assume 
$I=x R$ for some nonzero $x \in R$. Then $x R=I=y I=y x R$, so that $x=y x r$ for some $r \in R$. Then $x(1-y r)=0$, and since $y \in J, x=0$, a contradiction. Thus $I=x J$ for some nonzero $x \in R$. But if $x \in y^{n} R$ for every $n$, then $x R \cong I=x J$, and $x R \supsetneqq x J$ by Lemma 2.16, a contradiction. Hence, there must exist an integer $n$ such that $x R \supseteqq y^{n} R$. But then $x r=y^{n}$ for some $r \in R$, so $y x r=y^{n+1}$, and since $y x \in x J$, $y^{n+1} \in I$. This contradiction establishes that $I=0$ so that $J$ is nil.

CoRollary 2.21. A CLH ring $R$ is uniserial if and only if $J$ is a principal ideal.

Proof. Let $R$ be uniserial. Then every ideal is principal. Conversely, if $J$ is principal, then $J$ is nilpotent. Since $J^{n} / J^{n+1}$ is semi-simple for every $n$, it must be simple by the linear order on ideals. Consequently, if $p=$ index of nilpotency of $J$, then

$$
R \supset J \supset J^{2} \supset \cdots \supset J^{p-1} \supset 0
$$

is the unique composition series for $R$. Since $R$ is primary, $R$ is uniserial.

Lemma 2.22. Let $R$ be a commutative local ring satisfying the following:

(1) $J$ is the union of an ascending chain of proper principal subideals and is nil, but not nilpotent, $J=\bigcup_{1}^{\infty} x_{i} R$.

(2) Ideals in $R$ are linearly ordered.

(3) $S \neq 0$.

(4) Ideals in $R$ are of the form $x R$ or $x J$.

Then the following are true.

(a) $J=J^{2}$.

(b) For every $x \in R, x J$ is not finitely generated unless $x J=0$.

(c) $J^{2}=\left\{j_{1} j_{2} \mid j_{1}, j_{2} \in J\right\}$.

Proof. (a ) Look at $J / J^{2}$. Since $\left(J / J^{2}\right) J=0, J / J^{2}$ is either 0 or is simple. But if $x \in J$ is such that $x \notin J^{2}$, then $x R \nsupseteq J^{2}$, so that $x R=J$. But $J$ can't be principal since $J$ is not nilpotent. Thus $J / J^{2}=0$ so $J=J^{2}$.

(b) If $x J$ is finitely generated then $x J=z R$ for some $z \in R$. Then $z R=x J=x J^{2}=z R J=z J$, which is impossible unless $z R=0$.

(c) Clearly $A=\left\{j_{1} j_{2} \mid j_{1}, j_{2} \in J\right\} \subseteq J^{2}$. If

$$
z=\sum_{1=1}^{n} a_{i} b_{i} \in J^{2}, a_{i}, b_{i} \in J,
$$

then by the linear order on ideals, there is an integer $k$ such that 
$a_{i} b_{i} \in a_{k} b_{k} R, i=1, \cdots, n$. But then we can write $a_{i} b_{i}=a_{k} b_{k} r_{i}$, and $z=a_{k} b_{k} \sum_{1}^{n} r_{i}=a_{k}\left(b_{k} \sum_{1}^{n} r_{i}\right) \in A$.

THeOREM 2.23. Let $R$ satisfy the conditions of Lemma 2.22. Then we conclude

(a) Ideals in $R$ are annihilators.

(b) If $\varphi: x R \rightarrow R$ or $\varphi: x R \rightarrow R / S$, then $\varphi$ can be given by a left multiplication.

(c) If $I$ is an ideal in $R$, then $R / I$ embeds as an essential submodule of either $R$ orR/S.

(d) $(R / S)_{R}$ is injective if and only if $R_{R}$ is injective.

Proof. (a) Let $J=\bigcup_{1}^{\infty} x_{i} R$ where $x_{1} R=S, x_{i} R \varsubsetneqq x_{i+1} R$. Let $I=x J$. Since $S \subseteq x R$, there is $y \in R$ such that $0 \neq x y \in S$, and it follows that $x J=(0: y)$. If $I=x R$, again there is $y \in R$ such that $0 \neq x y \in S$. To show that $x R$ is the annihilator of $y J$, we need only observe that if $z \in(0: y J)$, then $z y J=0$ so that $z y \in S$. But if $z R \supseteqq x R$, then there is an element $r \in J$ such that $z r=x$. Then $x_{1}=x y=y z r=0$ since $y z \in S$ and $r \in J$. So $z \in x R$, and $x R=(0: y J)$.

(b) Let $\varphi: x R \rightarrow R$. Then $\varphi(x R)=\varphi(x) R$ is a principal ideal. Now $x r=0$ implies $\varphi(x r)=0$ so that $\varphi(x) r=0$. Hence, $r \in(0: x)$ implies $r \in(0: \varphi(x))$. However, if $(0: x) \subseteq(0: \varphi(x))$ then $\varphi(x) R \cong x R$, by taking annihilators, hence, there is an element $m \in R$ such that $\varphi(x)=m x$, and so $\varphi(x r)=m x r$ for all $x r \in x R$. Let $\varphi: x R \rightarrow R / S$. Again $\varphi(x R)=(y+S) R$ where $\varphi(x)=y+S$. But if $x r=0$, then $\varphi(x r)=0+S$, so $(0: x) \cong(S: y)$. Now $(0: x)=t J$ for some $t \in R$. Since $J^{2}=J$ by Lemma $2.22, t J^{2}=t J$. But $y t J \subseteq S$, so $y t J^{2} \subseteq S J=0$. Since $y t J^{2}=y t J,(0: x)=t J \subseteq(0: y)$. Hence, $y R \subseteq x R$, so $y=m x$. Then $\varphi(x r)=\varphi(x) r=(m x+S) r=(m+S) x r$.

(c) Let $I=x J$. Then there is a $y \in R$ such that $I=(0: y)$. Then $R / I \cong y R$ under $r+I \rightarrow y r$, so $R / I$ embeds in $R$.

Let $I=x R . \quad R / x R \cong R / x J / x R / x J$, and since $R / x J \cong y R$ as above $x R / x J \cong S, R / I \cong y R / S$. Thus $R / I$ embeds in $R / S$.

To see that the embeddings above are essential, one need only notice that if $m \in R$ or $R / S$, then $m R$ contains or is contained in the image of $R / I$, so that if $m R \cap \varphi(R / I)=0$ then $m R=0$.

(d) Notice that by virtue of (b), we need only show that maps of ideals of the form $x J$ are given by left multiplications in order to show that $R$ or $R / S$ satisfies Baer's condition. Also, if $\left\{x_{i}\right\}$ is the set of generators of the radical, then $\varphi$ is completely determined by its action on $\left\{x x_{i}\right\}$, that is, if we can show that $\varphi\left(x x_{i}\right)=m x x_{i}$ for every $i$, we will know that $\varphi$ is determined by left multiplication by $m$.

Now if $A$ is any ideal in $R$ with a maximal sub-ideal $B$, then by the linear order on ideals, $A=x R$ for any $x \in A$ such that $x \notin B$. On 
the other hand, any ideal of the form $x R$ has maximal submodule $x J$ as in Lemma 2.16, so that $A$ has a maximal submodule if and only if $A$ is principal (finitely generated, by the linear order on ideals). We see that the nonfinitely generated ideals in $R$ are precisely the ideals of the form $x J$ for any $x \in R, x \notin S$. Now since $x J$ is not finitely generated it has no maximal submodule, and consequently, its nonzero image under homomorphism to $R$ or $R / S$ cannot have a maximal submodule and so must be not finitely generated. Consequently, if $\varphi: x J \rightarrow R$, then $\varphi(x J)=y J$ for some $y \in R$, and if $\varphi: x J \rightarrow R / S$, $\varphi(x J)=y J / S$ for some $y \in R$.

Assume first that $R$ is injective, and let $\varphi: x J \rightarrow x J / S$ be an $R$-epimorphism. Then $\varphi_{i}=\left(\varphi\right.$ restricted to $\left.x x_{i} R\right)$ determines a map from $x x_{i} R$ into $y J / S$, and the maps $\varphi_{i}$ have the property that if $i \geqq j$, then $\varphi_{j}(r)=\varphi_{i}(r)$ for all $r$ in $x x_{j} R$. Since $x_{i} R \varsubsetneqq x_{i+1} R$, there is an element $t_{i} \in J$ such that $x_{i+1} t_{i}=x_{i}$. By (b), there exists $m_{i} \in R / S$ such that $\varphi_{i}\left(x x_{i} r\right)=m_{i} x x_{i} r$ for all $x x_{i} r \in x x_{i} R$. Let $m_{i}=j_{i}+S$. Then $\varphi\left(x x_{i} r\right)=\varphi_{i}\left(x x_{i} r\right)=m_{i} x x_{i} r$, so that $\varphi\left(x x_{i}\right)=x x_{i} j_{i}+S$. However, $x x_{i+1} t_{i}=x x_{i}$, so that

$$
\begin{aligned}
\varphi\left(x x_{i+1} t_{i}\right)=\varphi\left(x x_{i+1}\right) t_{i} & =\left(x x_{i+1} j_{i+1}+S\right) t_{i} \\
& =x x_{i+1} j_{i+1} t_{i}+S=x x_{i} j_{i+1}+S .
\end{aligned}
$$

Hence $x x_{i} j_{i}-x x_{i} j_{i+1}=x x_{i} r_{i} \in S$. We now define a new map from $x J$ into $R$ by giving its values on $\left\{x x_{i}\right\}_{\text {. Let }} \bar{\phi}\left(x x_{i}\right)=x x_{i} j_{i+1}$. Then $\bar{\varphi}$ will be well defined provided $\bar{\varphi}\left(x x_{i}\right)=\bar{\varphi}\left(x x_{i+1}\right) t_{i}$. But

$$
\begin{aligned}
\bar{\varphi}\left(x x_{i+1}\right) t_{i}=\left(x x_{i+1} j_{i+2}\right) t_{i} & =\left(x x_{i+1} j_{i+1}-x x_{i+1} r_{i+1}\right) t_{i} \\
& =x x_{i+1} j_{i+1} t_{i}-x x_{i+1} r_{i+1} t_{i}=x x_{i} j_{i+1},
\end{aligned}
$$

since $x_{i+1} t_{i}=x_{i+1}$ and $x x_{i+1} r_{i+1} \in S$ and $t_{i} \in J$. Thus $\bar{\varphi}$ is a well defined $R$-homomorphism of $x J$ into $R$. Since $R$ is injective, there is an $m \in R$ such that $\bar{\phi}(x j)=m x J$ for all $x j \in x J$. We assert that $\varphi(x j)=$ $(m+S) x j$ for all $x j \in x J$. Again, this will be true provided $\varphi\left(x x_{i}\right)=$ $(m+s) x x_{i}$ for every integer $i$. However, that $\varphi\left(x x_{i}\right)=x x_{i} j_{i}+S=$ $x x_{i} j_{i+1}+S$ was shown above, and $x x_{i} j_{i+1}=m x x_{i}$, so that $\varphi\left(x x_{i}\right)=$ $m x x_{i}+S=(m+S) x x_{i}$. Consequently $R / S$ is injective.

Conversely, assume that $R / S$ is injective, and let $\varphi: x J \rightarrow y J$ be an epimorphism. If $\nu$ denotes the natural map from $y J$ onto $y J / S$, then $\nu \varphi$ is a mapping from $x J$ onto $y J / S$. Since $R / S$ is injective, there is $z \in R$ such that $\nu \varphi(x j)=(z+S) x j$ for all $x j \in x J$. We show that the map $\varphi$ is defined by $\varphi(x j)=z x j$ for all $x j \in x J$. Since

$$
z x j+S=\nu \varphi(x j)=\varphi(x j)+S, \varphi(x j)-z x J \in S .
$$

Now $j \in J=J^{2}=\left\{j_{1} j_{2} \mid j_{1}, j_{2} \in J\right\}$ by Lemma 2.22 , so that there are $a, b \in J$ such that $j=a b$. Then 


$$
\varphi(x j)-z x j=\varphi(x a b)-z x a b=\varphi(x a) b-z x a b=(\varphi(x a)-z x a) b,
$$

and since $\varphi(x a)-z x a \in S$ and $b \in J,(\varphi(x a)-z x a) b=0$, so that $\varphi(x j)=$ $z x j$ for all $x j \in x J$. This completes the proof of (d), and thus of the theorem.

We now come to our main result.

THEOREM 2.24. Let $R$ be a commutative local ring. Then $R$ is $\mathrm{CLH}$ if and only if either (A) $R$ is uniserial, or (B) $R$ satisfies the following conditions:

(1) $J$ is the union of an ascending chain of proper principal subideals, and $J$ is nil.

(2) Ideals in $R$ are linearly ordered.

(3) $S \neq 0$.

(4) Ideals in $R$ are of the form $x R$ or $x J$.

(5) $R_{R}$ is injective.

Proof. Let $R$ be CLH, and assume $R$ is not uniserial. We have already shown that conditions (2) through (5) are satisfied using Theorem 2.7, Theorem 2.10, Theorem 2.19, and Corollary 2.2 respectively, hence we need only show (1). Let $\left\{x_{i}\right\}$ be a collection of elements of $J$ such that $x_{i}$ has index of nilpotence $n_{i}, n_{1}<n_{2}<n_{3} \ldots$. Such elements must exist since $J$ is nil by Theorem 2.20, and if $J$ were nilpotent, $R$ would be uniserial $\left(R \supset J \supset J^{2} \cdots \supset J^{2}=0\right.$ the unique composition series). We assert that $J=\bigcup_{1}^{\infty} x_{i} R$. Since $J$ is not principal by Theorem 2.21 , the ideals $x_{i} R$ are properly contained in $J$. Let $y \in J, n=$ index of nilpotency of $y$. Choose $i$ such that $n_{i}>n$. Then $y R \subseteq x_{i} R$ for if not, $x_{i} R \leqq y R$ by (2) and so $x=y r$, whence $x^{n}=y^{n} r^{n}=0$, contradicting the choice of $n_{i}$. Hence $J \subset \bigcup_{1}^{\infty} x_{i} R$ and so (1) is satisfied.

Conversely, since any uniserial ring is hypercyclic, we need only show that conditions (1)-(5) imply that $R$ is CLH. However, this is a trivial consequence of Theorem 2.23 .

We are now in a position to give an example of a nonartinian hypercyclic ring, which will show that the assumption of chain conditions was necessary in order to obtain the equivalence of hypercyclic and uniserial.

ExAMPle. Let $K$ be a field, $x$ an indeterminate over $K$, and let $W=\left\{\left\{\alpha_{i}\right\} \mid\left\{\alpha_{i}\right\}\right.$ a well ordered sequence of positive real numbers $\}$. Form the ring $T=\left\{\sum_{i=0}^{\infty} a_{i} x^{\alpha_{i}} \mid a_{i} \in K,\left\{\alpha_{i}\right\} \in W\right\}$. Then $T$ is a local ring, commutative, and $J(T)=\left\{\sum_{i=0} a_{i} x^{\alpha_{i}} \in T \mid \alpha_{0}>0\right\}$. Furthermore, if $r=\sum_{i=0}^{\infty} a_{i} x^{\alpha_{i}} \in J(T)$, and $\alpha_{k}$ is the minimal element of $\left\{\alpha_{i}\right\}$, then $r=$ $x^{\alpha_{k}} \sum_{0}^{\infty} a_{i} x^{\alpha_{i}-\alpha_{k}}$, and since $r^{\prime}=\sum_{i=0}^{\infty} a_{i} x^{\alpha_{i}-\alpha_{k}}=a_{k}+\sum_{i \neq k} a_{i} x^{\alpha_{i}-\alpha_{k}}, r^{\prime}$ is 
regular in $T$. Consequently, if $z, y \in J(T), z=x^{\alpha} r_{1}, y=x^{\beta} r_{2}$ with $r_{1}, r_{2}$ regular in $T$, and if $\alpha>\beta, z=x^{\alpha} r_{1}=x^{\beta} r_{2} x^{\alpha-\beta} r_{1} r_{2}^{-1}=y x^{\alpha-\beta} r_{1} r_{2}^{-1} \in y T$, so that $z T \subseteq y T$. Hence the ideals in $T$ are linearly ordered, and $z T / z J(T)$ is simple. Now let $I$ be any ideal in $J(T)$. Then the set $A=\left\{\alpha \in\right.$ Reals $\left.\mid x^{\alpha} \in I\right\}$ is a nonempty set of reals bounded below by 0 . Let $\alpha$ be the greatest lower bound of $A$. There are two cases:

Case 1. $\alpha \in A$. Then $x^{\alpha} \in I$, and if $\beta<\alpha$, then $x^{\beta} \notin I$, so that $x^{\beta} T \supsetneqq I$ by the linear order, and so $I=x^{\alpha} T$ is principal.

Case 2. $\alpha \notin A$. Then $x^{\alpha} \notin I$. But if $x^{\beta} \in I$, then $x^{\gamma} \in I$ for every $\gamma>\beta$, so that since $\alpha=$ g.l.b. $A, x^{\alpha+\beta} \in I$ for every $\beta>0$. Consequently, $x^{\alpha} J(T) \subseteq I$, and since $x^{\alpha} T / x^{\alpha} J(T)$ is simple and $x^{\alpha} T \supsetneqq I, I=x^{\alpha} J(T)$.

Let $R=T / x J(T)$. Then $R$ inherits the properties of having a linear order on ideals and having two kinds of ideals, $z R$ or $z J$. Furthermore, $R$ has simple socle $S=x T / x J(T)$ and $J(R)$ is nil, since if $x^{\alpha} r+x J(T) \in J(R)$ and $n \alpha>1$, then

$$
\left(x^{\alpha} r+x J(T)\right)^{n}=x^{n \alpha} r^{n}+x J(T)=0+x J(T) .
$$

Hence, in order to show that $R$ is a CLH ring, we need only show that $R$ is injective (or $R / S$ is injective). However, in [6] it is shown that every homomorphic image of $T$ is a self injective ring. Consequently, we have exhibited an example of a nonartinian CLH ring.

Notice that our example of a nonartinian CLH ring has the property that $R / S$ has no socle, and consequently cannot be a CLH ring itself. This raises the question of when are homomorphic rings of hypercyclic rings again hypercyclic.

THEOREM 2.25. Let $R$ be commutative. Then every homomorphic image of $R$ is a hypercyclic ring if and only if $R$ is uniserial.

Proof. Let $R$ be uniserial. Since every homomorphic image of a uniserial ring is uniserial and since every uniserial ring is hypercyclic, every homomorphic image of $R$ is hypercyclic.

Conversely, assume every homomorphic image of $R$ is hypercyclic. If $e R$ denotes one of the local components of $R$, and $e S$ denote its socle, $e R / e S$ must be a local hypercyclic ring, and must consequently have nonzero socle. By Theorem 2.12, eR/eS embeds in $e R$ so that $e J$ is principal. By Corollary 2.21, $e R$ is uniserial. Since $R$ is, then, a direct sum of uniserial rings, $R$ is uniserial and the proof is complete. 


\section{REFERENCES}

1. K. Asano, Uber verallgemeinerte Abelsche gruppen mit hypercomplexem Operatorenring und ihre Anwendungen, Japanese J. of Math. 15 (1939), 231-253.

2. H. Bass, Finististic dimension and a homological generalization of semi-primary rings, Trans. Amer. Math. Soc. 46 (1960), 466-488.

3. C. Faith, On Köthe rings, Mathematische Annalen 164 (1966), 207-212.

4. - Lectures on injective modules and quotient rings, Rutgers--The State University, New Brunswick, N. J. (1964), mimeographed.

5. M. Ikeda and T. Nakayama, On some characteristic properties of quasi-Frobenius and regular rings, Proc. Amer. Math. Soc. 5 (1954), 15-19.

6. L. Levy, Commutative rings whose homomorphic images are self injective (to appear)

7. B. Osofsky, Rings all of whose finitely generated modules are injective, Pacific J. Math. 14 (1964), 645-650.

8. A. Rosenberg and D. Zelinsky, Annihilators, Portugalia Mathematica 20 (1961), $53-65$.

9. - Finiteness of the injective hull, Mathematische Zeitschrift 70 (1959), $372-380$.

10. Y. Utumi, On continuous rings and self injective rings, Trans. Amer. Math. Soc. 118 (1965), 158-173.

Received December 8, 1966. This paper is a portion of the author's doctoral dissertation written at Rutgers-The State University under the direction of Professor Carl Faith.

Rutgers The State University and

UNIVERSITY OF SOUTh CAROLINA 


\section{PACIFIC JOURNAL OF MATHEMATICS}

\section{EDITORS}

\section{H. ROYDEN}

Stanford University

Stanford, California

\section{J. P. Jans}

University of Washington

Seattle, Washington 98105

\section{J. DUGUNDJI}

Department of Mathematics

Rice University

Houston, Texas 77001

RICHARD ARENS

University of California

Los Angeles, California 90024

\section{ASSOCIATE EDITORS}
E. F. BeCKenbaCH
B. H. NeumanN
F. WOLF
K. YosIDA

\section{SUPPORTING INSTITUTIONS}

UNIVERSITY OF BRITISH COLUMBIA CALIFORNIA INSTITUTE OF TECHNOLOGY UNIVERSITY OF CALIFORNIA MONTANA STATE UNIVERSITY UNIVERSITY OF NEVADA NEW MEXICO STATE UNIVERSITY OREGON STATE UNIVERSITY UNIVERSITY OF OREGON OSAKA UNIVERSITY UNIVERSITY OF SOUTHERN CALIFORNIA
STANFORD UNIVERSITY UNIVERSITY OF TOKYO UNIVERSITY OF UTAH WASHINGTON STATE UNIVERSITY UNIVERSITY OF WASHINGTON

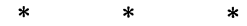

AMERICAN MATHEMATICAL SOCIETY CHEVRON RESEARCH CORPORATION TRW SYSTEMS

NAVAL ORDNANCE TEST STATION 


\section{Pacific Journal of Mathematics}

\section{Vol. 24, No. $1 \quad$ May, 1968}

Harry P. Allen, Lie algebras of type $D_{4}$ over algebraic number fields ...... 1

Charles Ballantine, Products of positive definite matrices. II............ 7

David W. Boyd, The spectral radius of averaging operators ............ 19

William Howard Caldwell, Hypercyclic rings ................... 29

Francis William Carroll, Some properties of sequences, with an application

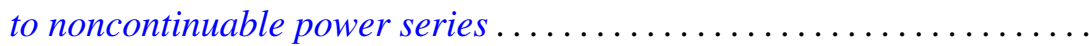

David Fleming Dawson, Matrix summability over certain classes of

sequences ordered with respect to rate of convergence ........... 51

D. W. Dubois, Second note on David Harrison's theory of preprimes. . . . . 57

Edgar Earle Enochs, A note on quasi-Frobenius rings.............. 69

Ronald J. Ensey, Isomorphism invariants for Abelian groups modulo bounded groups ................................ 71

Ronald Owen Fulp, Generalized semigroup kernels ................ 93

Bernard Robert Kripke and Richard Bruce Holmes, Interposition and approximation ................................. 103

Jack W. Macki and James Sai-Wing Wong, Oscillation of solutions to second-order nonlinear differential equations ..................

Lothrop Mittenthal, Operator valued analytic functions and generalizations

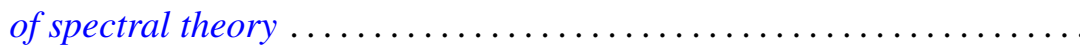

T. S. Motzkin and J. L. Walsh, A persistent local maximum of the pth power deviation on an interval, $p<1 \ldots \ldots \ldots \ldots \ldots \ldots \ldots \ldots \ldots \ldots . \ldots \ldots$

Jerome L. Paul, Sequences of homeomorphisms which converge to homeomorphisms ...........................

Maxwell Alexander Rosenlicht, Liouville's theorem on functions with elementary integrals.

Joseph Goeffrey Rosenstein, Initial segments of degrees .

$\mathrm{H}$. Subramanian, Ideal neighbourhoods in a ring ............

Dalton Tarwater, Galois cohomology of abelian groups . .

James Patrick Williams, Schwarz norms for operators ... .

Raymond Y. T. Wong, A wild Cantor set in the Hilbert cube. 